\title{
Radiocesium in Fish from the Savannah River and Steel Creek: Potential Food Chain Exposure to the Public
}

\author{
Joanna Burger, ${ }^{1,2, *}$ K. F. Gaines, ${ }^{3}$ J. D. Peles, ${ }^{4}$ W. L. Stephens Jr., ${ }^{3}$ C. Shane Boring, ${ }^{1,2}$ \\ I. L. Brisbin Jr., ${ }^{3}$ J. Snodgrass, ${ }^{1,2,5}$ A. L. Bryan, Jr., ${ }^{3}$ M. H. Smith, ${ }^{3}$ and M. Gochfeld ${ }^{2,6}$
}

\begin{abstract}
This study examined radiocesium $\left({ }^{137} \mathrm{Cs}\right)$ levels in fish from the vicinity of the Department of Energy's Savannah River Site (SRS), a former nuclear weapons production facility in South Carolina. Fish from the Savannah River were sampled above (upstream), along, and below (downstream) the SRS, and from Steel Creek, a tributary that runs through the SRS. There was some off-site contamination of ${ }^{137} \mathrm{Cs}$ in the Savannah River watershed due to low-level releases from past nuclear production on the SRS. The null hypotheses tested were that there would be no differences in ${ }^{137} \mathrm{Cs}$ levels as a function of location along the river, and between species collected from the river and from Steel Creek on the SRS. For six of eight species of fish collected from the Savannah River, there were no differences in ${ }^{137} \mathrm{Cs}$ levels in muscle from fish collected above, along, or below the SRS; exceptions were bowfin and shellcracker. Fish collected from Steel Creek had significantly higher levels (by about an order of magnitude) of ${ }^{137} \mathrm{Cs}$ in muscle tissue than fish collected in the Savannah River. However, no fish from either Steel Creek or the Savannah River had ${ }^{137} \mathrm{Cs}$ levels above the European Economic Community limit for fresh meat of $0.6 \mathrm{~Bq} / \mathrm{g}$. Lifetime cancer risk was calculated using the cancer slope factor of $3.2 \times 10^{-11} / \mathrm{pCi}$, and various fish consumption scenarios reflecting actual data from Savannah River fishermen. Using mean ${ }^{137} \mathrm{Cs}$ concentrations and median fish consumption for 70 years for Black males-the group with the highest consumption-the excess lifetime risk associated with the eight species of fish in the Savannah River ranged from $9.0 \times 10^{-7}$ to $1.0 \times 10^{-5}$. The same calculation for fish from Steel Creek gave risk estimates from 1.4 to $8.0 \times 10^{-5}$. The $95 \%$ level for consumption by Blacks, however, was about $70 \mathrm{~kg} /$ year. Black fishermen consuming that amount of bass from Steel Creek would sustain a lifetime risk of $3.1 \times 10^{-4}$, whereas the same consumption of Savannah River bass would yield a risk estimate of $1.5 \times 10^{-5}$.
\end{abstract}

KEY WORDS: Radiocesium; fish; food chain; risk; predators; environmental hazards; fish consumption

${ }^{1}$ Division of Life Sciences, Nelson Hall, Rutgers University, Piscataway, NJ.

${ }^{2}$ Consortium for Risk Evaluation with Stakeholder Participation, Environmental and Occupational Health Sciences Institute, Piscataway, NJ.

${ }^{3}$ Savannah River Ecology Laboratory, Aiken, SC.

${ }^{4}$ Pennsylvania State University-McKeesport, McKeesport, PA.

${ }^{5}$ Department of Biology, Towson University, Towson, MD.

${ }^{6}$ Environmental and Community Medicine, UMDNJ-Robert Wood Johnson Medical School, Piscataway, NJ.

*Address correspondence to Joanna Burger, Division of Life Sciences, 604 Allison Road, Rutgers University, Piscataway, NJ 088548082; burger@biology.rutgers.edu.

\section{INTRODUCTION}

For protection of viable populations of all living organisms, including humans, it is desirable to assess, avoid, and mitigate potential environmental hazards. Scientists have devoted considerable time to assessing the hazards posed to organisms by metals, organochlorines, other pesticides, and radionuclides. ${ }^{(1-6)}$ Understanding the transport, fate, and effects of contaminants is particularly critical in aquatic systems because of the potential for rapid movement through 
the water medium, and the rapid accumulation up the food chain. In addition, many aquatic organisms, such as fish, move considerable distances within stream systems, carrying contaminants with them. Fish are particularly useful as bioindicators of contamination because of their role in the food chain, both for humans and other organisms. The potential for adverse effects from contaminants is particularly strong near industrial sites, including chemical and nuclear power or weapons production facilities. ${ }^{(7,8)}$

This article examines ${ }^{137} \mathrm{Cs}$ levels in the muscle (fillets) of nine species of fish from the vicinity of the Savannah River Site (SRS), a former nuclear weapons production facility and current research facility in South Carolina, operated by the U.S. Department of Energy. Fish were collected from the Savannah River upstream of the SRS (above), from the section of river bordering the SRS (along), and downstream of the SRS (below). Fish species were selected to represent different levels of the food chain, and to include species eaten by the local fishermen. We also report ${ }^{137} \mathrm{Cs}$ levels in seven of these species collected from Steel Creek, a tributary of the Savannah River that is found inside the SRS site boundaries, drains into the Savannah River, and is known to have elevated levels of ${ }^{137} \mathrm{Cs}$. During SRS's years of nuclear weapons production, a variety of chemicals and radioactive wastes were released through emissions and disposal activities, and some water bodies received much contamination.

Our overall objective was to assess potential food chain effects, including those on humans consuming fish. South Carolina and Georgia have issued fish consumption advisories for the Savannah River, based on mercury and radionuclides. ${ }^{(9)}$ The public is not allowed on the SRS itself to fish, although some illegal fishing does occur on-site. ${ }^{(10)}$ This research is part of a larger project to assess the potential for human and ecological effects from off-site contamination from the SRS, ${ }^{(10-12)}$ and, therefore, levels of heavy metals are considered elsewhere.

Radiocesium is widespread in soils of the northern hemisphere due to fallout from above-ground nuclear weapons testing, and contamination is greatly affected by soil type; it was released to both air and to water. This background contamination, which peaked in the mid-1960s, is not completely homogeneous, and varies more with latitude than longitude. ${ }^{(13)} \mathrm{Su}-$ perimposed on that level are point-source releases such as those from the nuclear reactors, chemical separation facilities, and other activities on the SRS. Airborne Cs deposits on soil and percolates downward with rainwater. Radiocesium has a radioactive half-life of about 30 years, and it is slowly mobilized from sediments. ${ }^{(14)}$

Studies of chemical cycling, including fate and transport, have provided models for understanding the role of ${ }^{137} \mathrm{Cs}$ in ecosystems. ${ }^{(15-17)}{ }^{137} \mathrm{Cs}$ accumulates in muscle ${ }^{(17-19)}$ and thus is available to predators (including humans) that consume any contaminated animals. It is especially important to understand the levels of ${ }^{137} \mathrm{Cs}$ in fish in rivers and streams because fish can move over large distances during routine movements and migrations, and are eaten by many different organisms, including humans.

Much of the research on ${ }^{137} \mathrm{Cs}$ has been conducted in lakes and streams associated with nuclear reactors, ${ }^{(20,21)}$ on atolls exposed to nuclear tests, ${ }^{(22)}$ or in response to the 1986 Chernobyl accident. ${ }^{(23-25)}$ To date, insufficient attention has been devoted to ${ }^{137} \mathrm{Cs}$ in tissue of fish from rivers or streams adjacent to industrial and nuclear facilities, where bioaccumulation might pose a threat to the food chain, including human consumers. State and federal agencies often conduct analyses for contaminants, but seldom include radionuclides. ${ }^{(9,26)}$ Such routine monitoring results are rarely reported in the literature, and as a result, there is frequently insufficient information to conduct exposure models of the transfer of these radionuclides to the public through recreational and subsistence fishing, and to adequately assess lifetime cancer risk from radionuclides.

Understanding contamination of fish in the Savannah River is particularly important because it is a relatively large river with a diverse fish assemblage; the fish from the river are available to a wide range of predators, including endangered and threatened birds; and fishing plays an important role in the lives of local people. ${ }^{(27)}$ Recently, Burger ${ }^{(10,11)}$ surveyed fishermen along the Savannah River and reported (1) a wide range in consumption rates, (2) that some fishermen consumed more than the amount used by the U.S. Environmental Protection Agency (USEPA) to compute the risk to recreational and subsistence fishermen, and (3) ethnic differences in fish consumption rates (Blacks consumed significantly more fish per year than Whites). Current information on radiocesium levels, along with other contaminants such as mercury, is essential to determine the risk that these fishermen and their families face from fish consumption.

This study had several unique features compared with other risk assessments on ${ }^{137} \mathrm{Cs}$, including trophic differences in contamination levels, tissue level data from fish collected above and below the SRS (i.e., locational differences), risk scenarios based on sitespecific consumption patterns, and separate risk sce- 
narios for Black and White fishermen. Finally, the data set was large enough and sufficiently diverse to examine the relation between fish size and ${ }^{137} \mathrm{Cs}$ levels, allowing for possible risk reduction by selecting smaller fish for consumption.

\section{STUDY AREA AND METHODS}

\subsection{Study Areas}

The study was conducted on fish from the Savannah River, which forms the southern boundary of the Department of Energy's SRS in South Carolina. The overall study design was to collect fish from above, along, and below the SRS in 1997, and from nearby Steel Creek in 1998 (see Fig. 1). We defined "above" as the stretch of river from the Augusta Lock and Dam to the north edge of the SRS. "Along" was the stretch of river bordering the site. "Below" extended from the south edge of the SRS to the Route 301 bridge across the Savannah River (Fig. 1).

The SRS, located in west central South Carolina $\left(33.1^{\circ} \mathrm{N}, 81.3^{\circ} \mathrm{W}\right)$, is a $780-\mathrm{km}^{2}$ former nuclear weapons production and current research facility operated by the U.S. government since the early 1950s. The SRS is bounded on the south by the Savannah River, which was used as a source of cooling water for five reactors when they were functioning. The Savannah

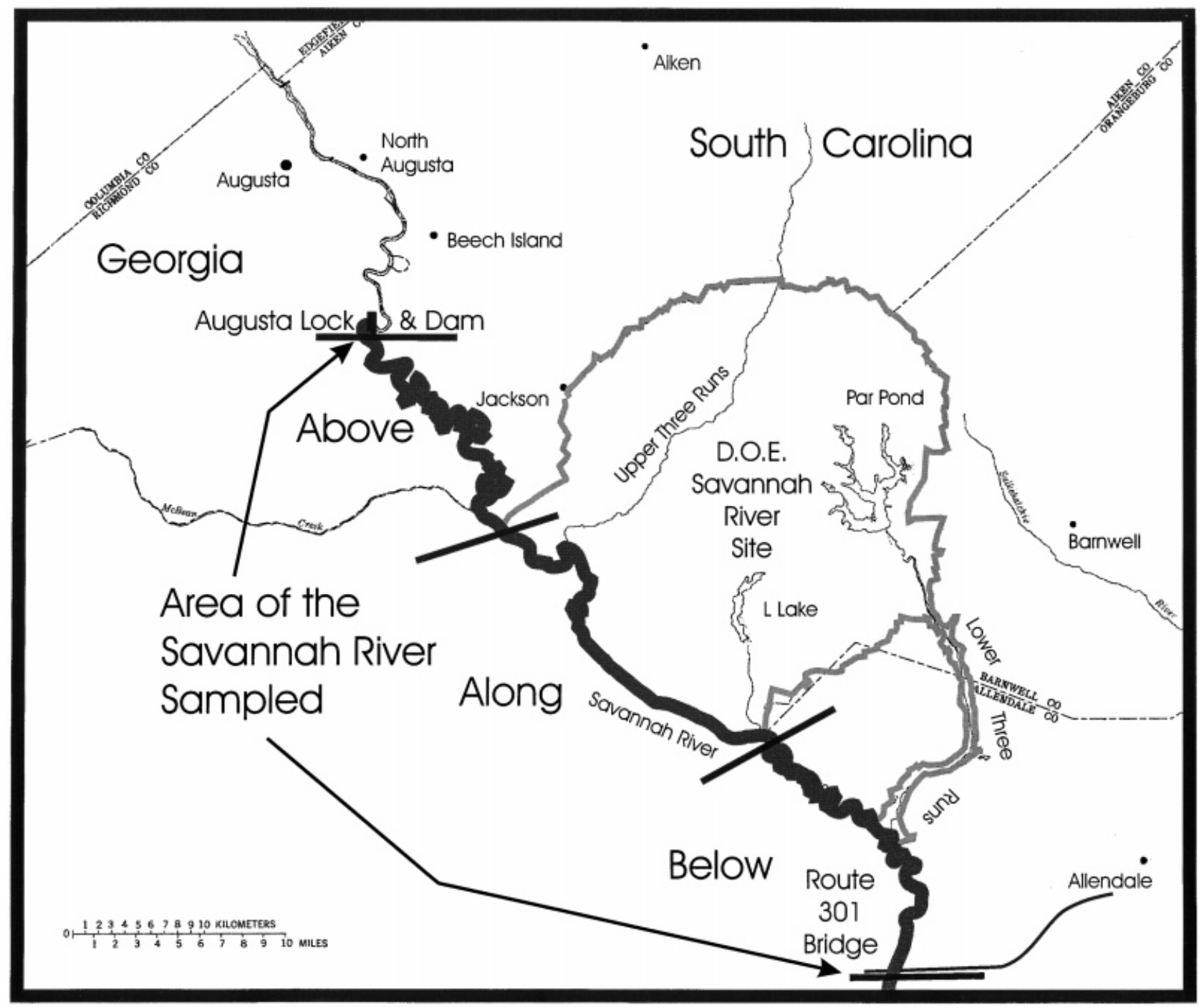

Fig. 1. Map of the Savannah River Site (SRS), showing locations of fish collection above, along, and below the SRS, Steel Creek (below L Lake to the river), and Par Pond. D.O.E. = Department of Energy. 
River separates Georgia from South Carolina, and flows into the Atlantic Ocean. Upper Coastal Plain plant communities dominate the SRS, including pine forests, sandhill forests, freshwater streams, and bottomland swamps. ${ }^{(28)}$ Streams from the SRS flow directly into the Savannah River, and there are neither natural nor artificial barriers to prevent fish from moving between the site and the river.

The SRS produced plutonium and tritium and processed other nuclear materials for national defense and other governmental and private industrial purposes. Radiocesium was released into the environment during the operation of a high-level waste storage system, two radiochemical processing facilities, and five production reactors. ${ }^{(29,30)}$ Carlton et al $^{(30)}$ estimated that $65 \%$ remained on-site. Impoundments on the SRS were used as thermal cooling reservoirs for nuclear production reactors. Prior to the construction of the cooling ponds, there was some ecosystem contamination of streams and the floodplain, and small quantities of radionuclides were released subsequently..$^{(8,17,31)}$

Steel Creek is a $20-\mathrm{km}$-long stream that, along with two smaller, parallel streams, drains a $290-\mathrm{km}^{2}$ watershed in Barnwell County, South Carolina. In its lower reaches, the flow of Steel Creek is partially dammed by levee deposits of the Savannah River, creating a river swamp known as the Steel Creek Delta. The majority of the swamp enters the Savannah River through a narrow channel directly southeast of the delta. From 1954 to 1968 Steel Creek received effluents from two nuclear production reactors along with purge water from reactor disassembly basins, resulting in the release of approximately $9.66 \times 10^{13} \mathrm{~Bq}$ of ${ }^{137} \mathrm{Cs}$ into this stream. Leakage of ${ }^{137} \mathrm{Cs}$ continued until 1978. ${ }^{(31)}$ Thus, although there is no new source of ${ }^{137} \mathrm{Cs}$, there is resuspension and cycling from upstream sediments and from sediments within Steel Creek itself.

\subsection{Fish Collection and Sample Preparation}

The research protocol was to collect 15 fish of each species from above, along, and below the SRS, and to collect fish from Steel Creek (see below) in 1998. All fish were collected under appropriate collecting permits from the state of South Carolina, and with protocol approvals from the University of Georgia Institutional Animal Care and Use Committee (A960205) and Rutgers University Institutional Review Board (07-017). Fish from the Savannah River were collected from a 6-m Smith Root Electrofisher boat; stunned fish were collected with dip nets, placed on ice, and returned to the Savannah River Ecology Laboratory (SREL). Species of fish collected included species at all trophic levels. (See Table I for species, scientific names, and trophic level, after Rohde et al. ${ }^{(32)}$ )

Fish from Steel Creek were collected by trapping, electrofishing, and angling (depending on the fish species) from two locations in the Steel Creek watershed during January and February, March through June, and September and October of 1998. The main stream channel was sampled along its lower reaches $(3.5 \mathrm{~km})$ between U.S. highway 125 and the entry of the stream channel into the Steel Creek Delta. Samples were also collected from a $2-\mathrm{km}^{2}$ area in the Steel Creek Delta. This area is $1.5 \mathrm{~km}$ from the entry of Steel Creek into the Savannah River and was separated from the nearest sampling point in the Steel Creek corridor by about $0.5 \mathrm{~km}$. All samples were washed free of debris and frozen until analysis.

Table I. Scientific Names, Diet, and Relative Trophic-Level Ranking of Fish Collected in the Savannah River

\begin{tabular}{|c|c|c|c|c|}
\hline Common name & Scientific name & Diet & $\begin{array}{l}\text { Trophic } \\
\text { ranking }\end{array}$ & Mobility \\
\hline Bowfin & Amia calva & Fish & 1 & Mobile \\
\hline Largemouth bass & Micropterus salmoides & Fish & 1 & Mobile \\
\hline Channel catfish & Ictalurus punctatus & Large invert/fish & 2 & Mobile \\
\hline Black crappie & Pomoxis nigromaculatus & Large invert/small fish & 3 & Highly mobile \\
\hline Yellow perch & Perca flavescens & Large invert/small fish & 3 & Sedentary \\
\hline Shellcracker $^{a}$ & Lepomis microlophus & Medium-large invert & 4 & Sedentary \\
\hline Red-breasted sunfish & Lepomis auritus & Medium-large invert & 4 & Sedentary \\
\hline Bluegill & Lepomis macrochirus & Medium-large invert & 4 & Sedentary \\
\hline Spotted sucker & Minytrema melanops & Detritus & 5 & Migratory \\
\hline
\end{tabular}

Note: A ranking of 1 represents a high-level piscivore $(1=$ highest $)$. After Rohde et al..$^{(32)}$ and Bennett and McFarlane. ${ }^{(40)}$

${ }^{a}$ Local designation. Standard common name is redear sunfish. 
In this study, data from fish from all locations in Steel Creek and the Steel Creek Delta were combined.

At the laboratory, fish were labeled by date and collection location and were frozen $\left(-4^{\circ} \mathrm{C}\right)$ for later dissection. During dissection at SREL, fish were weighed, their standard and total lengths were measured, and edible fillets were removed. ${ }^{137} \mathrm{Cs}$ levels of fillets were counted at SREL, and the samples were subsequently transported to the Environmental and Occupational Health Sciences Institute for metal analysis.

\subsection{Cesium Analysis}

The ${ }^{137} \mathrm{Cs}$ count rates of wet muscle fillets were determined using a Gamma-X HPGe high-purity germanium coaxial photon detector system with a $56.7 \times$ $77.3 \mathrm{~mm}$ crystal. An EG\&G Ortech 92x Spectrum Master integrated spectroscopy system with associated Gamma Vision software was used for data acquisition. A counting window of approximately 658-666 kiloelectron volts $(\mathrm{keV})$ was used after a peak region of interest was identified from calibration with a known ${ }^{137} \mathrm{Cs}$ standard to record total absorptions from the ${ }^{137} \mathrm{Cs}$ emission of $662 \mathrm{keV}$ photons. Prior to acquiring the Gamma-X HPGe system, ${ }^{137} \mathrm{Cs}$ levels for some larger fish were quantified using a $10.2 \times 15.2 \mathrm{NaI}$ detector coupled to a computer-based Canberra spectroscopy system (AccuSpec, Canberra Industries, Meriden, Connecticut, USA) that used a counting window of 596 to $728 \mathrm{keV}$ to record total absorptions from the $662 \mathrm{keV}$ photons. After measuring a subset of these samples in both systems, no significant difference was found based on a paired two-sample analysis. Both systems were calibrated prior to every counting sequence using a certified calibration standard. Counting time per sample was $120 \mathrm{~min}$, and 60-min background counts were performed immediately before and after each sample count to obtain the net count for each sample. To estimate concentrations of ${ }^{137} \mathrm{Cs}(\mathrm{Bq} / \mathrm{g}$ of wet tissue), we first adjusted sample gross count rates for the background count rates, then compared the adjusted count rates of aqueous ${ }^{137} \mathrm{Cs}$ standards approximating the sample geometry. Count rates of standards were also determined before or after every counting sequence (daily for the Canberra system and weekly for the Gamma-X HPGe system). Minimum detectable levels (MDLs) were calculated following the procedure of Currie. ${ }^{(33)}$

${ }^{137} \mathrm{Cs}$ counts for a random sample of 57 fish fillets were conducted at the Elemental Analysis Laboratory of the Environmental and Occupational Health Sci- ences Institute for comparison with those from SREL, using a High-Purity Germanium Gamma Spectroscopy system with digital ADC. The agreement between the two was high $(r=0.92, p<0.0001)$, and there were no significant differences between the samples.

\subsection{Statistical Analysis and Risk Assessment}

${ }^{137} \mathrm{Cs}$ distributions were examined using ShapiroWilk statistics (PROC UNIVARIATE, version 8.1; SAS Institute, 2000). Tests of hypotheses that these data were random samples from normal distributions and tests of homogeneity of variances were rejected $(p<0.05)$ and stem-and-leaf plots suggested a log transformation of data prior to analysis. Differences in ${ }^{137} \mathrm{Cs}$ among sites were then examined via ANOVA models (PROC GLM; SAS Institute, 1998). For all tested models, Type III (partial) sums of squares and associated $\mathrm{F}$ statistics were interpreted and leastsquare means (LS means) procedures were used to provide estimates of dependent variables that were adjusted for all effects in the models and to provide mean separation tests. All statistical tests were considered significant at $p \leq 0.05$, and Bonferroni corrections were performed when appropriate. Means and standard errors are presented as back-transformed values of $\log$ LS means estimates (i.e., geometric means). A Pearson correlation was used to determine the relation between ${ }^{137} \mathrm{Cs}$ and the fresh body mass of fish (log transformed).

Fish consumption data were obtained by interviewing fishermen at various points along the river (data from Burger et al. $\left.{ }^{(10)}\right)$. Data were obtained separately for Black and White males; risk for females was not computed because few women were interviewed in the study of fish consumption. ${ }^{11}$

To estimate the risk from fish ingestion, it was assumed that a fisherman would consume only one species throughout the year. The arithmetic mean concentration of cesium (Bq/g converted to $\mathrm{pCi} / \mathrm{g}$ ) was used for that species, and the amount of $\mathrm{pCi}$ of ${ }^{137} \mathrm{Cs}$ consumed in a year was estimated. Because most species showed no difference in Cs concentrations, all the Savannah River values for each species were pooled. The consumption distribution for Black and White males was used for the risk estimates.

For each fish, therefore, a range of $\mathrm{pCi} / \mathrm{yr}$ ingestions was obtained separately for the Savannah River and for Steel Creek. It was assumed that fish consumption from Steel Creek might be the same as the data obtained by interviews along the Savannah River; however, fishing is not actually allowed along 
Table II. Levels of Radiocesium (Fresh Weight of Muscle in Fish Fillet) Collected along the Savannah River Site (SRS)

\begin{tabular}{lccc}
\hline & \multicolumn{3}{c}{ Radiocesium level (Bg/g, wet weight) } \\
\cline { 2 - 4 } & Above SRS & Along SRS & Below SRS \\
\hline Bowfin $^{a}$ & $0.0055 / 0.013$ & $0.0095 / 0.066$ & $0.002 / 0.011$ \\
& $(n=16) \mathrm{B}$ & $(n=30) \mathrm{A}$ & $(n=13) \mathrm{B}$ \\
Largemouth bass & $0.002 / 0.016$ & $0.004 / 0.024$ & $0.002 / 0.008$ \\
& $(n=15)$ & $(n=15)$ & $(n=14)$ \\
Channel catfish & $0.0045 / 0.017$ & $0.0025 / 0.018$ & $0.002 / 0.016$ \\
& $(n=10)$ & $(n=22)$ & $(n=15)$ \\
Black crappie & $0^{b} / 0.011$ & $0.004 / 0.023$ & $0.003 / 0.011$ \\
& $(n=15)$ & $(n=35)$ & $(n=4)$ \\
Yellow perch & $0^{b} / 0.013$ & $0.004 / 0.031$ & $0.001 / 0.037$ \\
Shellcracker ${ }^{a, c}$ & $(n=8)$ & $(n=29)$ & $(n=17)$ \\
& $0.0085 / 0.027$ & $0.0025 / 0.013$ & $0^{b} / 0.012$ \\
Red-breasted sunfish & $(n=14) \mathrm{A}$ & $(n=14) \mathrm{AB}$ & $(n=16) \mathrm{B}$ \\
& $0.002 / 0.012$ & $0.003 / 0.014$ & none obtained \\
Bluegill & $(n=3)$ & $(n=24)$ & \\
& $0^{b} / 0.11$ & $0.0005 / 0.023$ & $0^{b} / 0.005$ \\
& $(n=20)$ & $(n=28)$ & $(n=12)$ \\
\hline
\end{tabular}

Note: Values given are median/maximum.

${ }^{a}$ Different letters denote significant differences ( $\chi^{2}$ tests) among sites for bowfin and shellcracker. Other species showed no significant difference between sites.

${ }^{b}$ All samples below MDC (minimum detectable concentration).

${ }^{c}$ Local designation. Standard common name is redear sunfish.
Steel Creek, although fish in the creek had access to the River and vice versa. Using the USEPA Cancer Assessment Group's slope factor of $3.2 \times 10^{-11}$ excess cancers per $\mathrm{pCi}$ of ${ }^{137} \mathrm{Cs}$ (EPA Integrated Risk Information System [IRIS] database; http://www.epa.gov/ iris), the excess cancer risk from a 1-year exposure was computed. We then assumed that a fisherman might consume fish over a 70-year period, and multiplied the annual risk increment by this value to obtain a lifetime risk estimate. Some older local fishermen indicated that they had spent 70 years fishing along the Savannah River. ${ }^{(10)}$

\section{RESULTS}

\subsection{Locational Differences in Fish from the Savannah River}

Except for bowfin and shellcracker, there were no significant differences in the levels of ${ }^{137} \mathrm{Cs}$ in the muscle (fillets) of fish from the three segments of the Savannah River, compared within species (Table II). The level of ${ }^{137} \mathrm{Cs}$ was significantly higher in bowfin captured along the SRS compared with either above or below the SRS, and in shellcracker collected above the SRS compared with below the SRS (Table II).

Table III. Radiocesium in Fish from the Savannah River and Steel Creek

\begin{tabular}{|c|c|c|c|c|c|c|c|c|}
\hline & & $N$ & Median & $\begin{array}{c}\text { (Bg/g, wet weight) } \\
M \pm S E\end{array}$ & $\begin{array}{c}\text { 75th } \\
\text { percentile }\end{array}$ & $\begin{array}{c}\text { 100th } \\
\text { percentile }\end{array}$ & $\begin{array}{l}\text { Correlation }^{a} \\
\text { with fresh body } \\
\text { mass of fish }\end{array}$ & $\begin{array}{c}\text { One-way } \\
\text { ANOVA, } F(p)\end{array}$ \\
\hline Bowfin & $\begin{array}{l}\text { River } \\
\text { Steel Creek }\end{array}$ & $\begin{array}{r}59 \\
6\end{array}$ & $\begin{array}{l}0.01 \\
0.04\end{array}$ & $\begin{array}{l}0.01 \pm 0.01 \\
0.06 \pm 0.06\end{array}$ & $\begin{array}{l}0.01 \\
0.12\end{array}$ & $\begin{array}{l}0.07 \\
0.14\end{array}$ & $-0.30(0.02)$ & $26.7(0.0001)$ \\
\hline Largemouth Bass & $\begin{array}{l}\text { River } \\
\text { Steel Creek }\end{array}$ & $\begin{array}{l}44 \\
34\end{array}$ & $\begin{array}{l}0.003 \\
0.07\end{array}$ & $\begin{array}{r}0.004 \pm 0.01 \\
0.08 \pm 0.05\end{array}$ & $\begin{array}{l}0.01 \\
0.10\end{array}$ & $\begin{array}{l}0.02 \\
0.19\end{array}$ & $-0.13(N S)$ & $198(0.0001)$ \\
\hline Channel catfish & $\begin{array}{l}\text { River } \\
\text { Steel Creek }\end{array}$ & $\begin{array}{r}47 \\
7\end{array}$ & $\begin{array}{l}0.003 \\
0.07\end{array}$ & $\begin{array}{r}0.004 \pm 0.01 \\
0.07 \pm 0.05\end{array}$ & $\begin{array}{l}0.01 \\
0.11\end{array}$ & $\begin{array}{l}0.02 \\
0.14\end{array}$ & $0.27(0.07)$ & $75.9(0.0001)$ \\
\hline Black crappie & $\begin{array}{l}\text { River } \\
\text { Steel Creek }\end{array}$ & $\begin{array}{r}54 \\
4\end{array}$ & $\begin{array}{l}0.003 \\
0.01\end{array}$ & $\begin{aligned} 0.003 & \pm 0.01 \\
0.01 & \pm 0.001\end{aligned}$ & $\begin{array}{l}0.01 \\
0.02\end{array}$ & $\begin{array}{l}0.02 \\
0.02\end{array}$ & $0.02(N S)$ & $5.20(0.03)$ \\
\hline Yellow perch & $\begin{array}{l}\text { River } \\
\text { Steel Creek }\end{array}$ & $\begin{array}{r}54 \\
6\end{array}$ & $\begin{array}{l}0.002 \\
0.04\end{array}$ & $\begin{array}{r}0.004 \pm 0.01 \\
0.05 \pm 0.05\end{array}$ & $\begin{array}{l}0.01 \\
0.06\end{array}$ & $\begin{array}{l}0.04 \\
0.14\end{array}$ & $0.12(N S)$ & $33.8(0.0001)$ \\
\hline Shellcracker ${ }^{c}$ & River & 44 & 0.002 & $0.003 \pm 0.01$ & 0.01 & 0.03 & $0.28(0.07)$ & $b$ \\
\hline Red-breasted sunfish & $\begin{array}{l}\text { River } \\
\text { Steel Creek }\end{array}$ & $\begin{array}{l}37 \\
32\end{array}$ & $\begin{array}{l}0.002 \\
0.05\end{array}$ & $\begin{aligned} 0.002 & \pm 0.01 \\
0.07 & \pm 0.04\end{aligned}$ & $\begin{array}{l}0.01 \\
0.10\end{array}$ & $\begin{array}{l}0.01 \\
0.18\end{array}$ & $0.095(N S)$ & $181(0.0001)$ \\
\hline Bluegill & River & 60 & 0.0007 & $0.001 \pm 0.001$ & 0.004 & 0.02 & $0.218(N S)$ & $b$ \\
\hline Spotted sucker & $\begin{array}{l}\text { River } \\
\text { Steel Creek }\end{array}$ & $\begin{array}{l}37 \\
26\end{array}$ & $\begin{array}{l}0.002 \\
0.02\end{array}$ & $\begin{aligned} 0.002 & \pm 0.003 \\
0.02 & \pm 0.06\end{aligned}$ & $\begin{array}{l}0.003 \\
0.03\end{array}$ & $\begin{array}{l}0.01 \\
0.12\end{array}$ & $0.031(N S)$ & $70.0(0.0001)$ \\
\hline
\end{tabular}

${ }^{a}$ For fish from the river only.

${ }^{b}$ No data available for Steel Creek.

${ }^{c}$ Local designation. Standard common name is redear sunfish. 

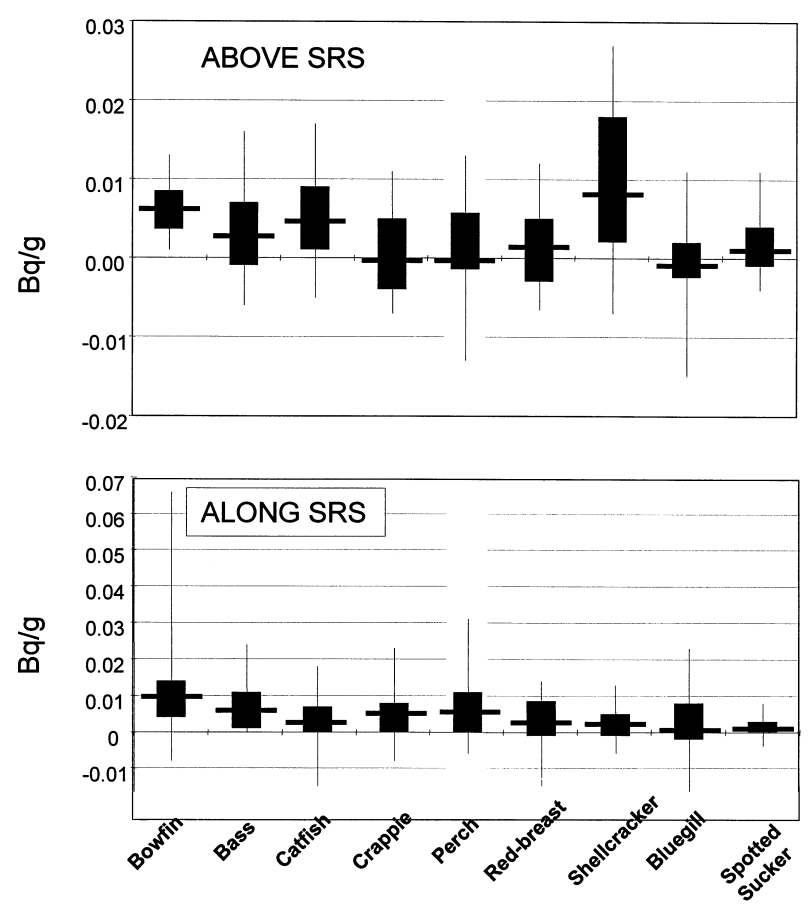

\subsection{Differences between Fish from the River and Steel Creek}

Two species, shellcracker and bluegill, were not obtained from Steel Creek. For the other species, there were significantly higher muscle levels of ${ }^{137} \mathrm{Cs}$ in fish collected from Steel Creek compared with fish collected from the Savannah River (Table III). Generally the levels in fish from Steel Creek were an order of magnitude higher than those in the fish from the Savannah River.

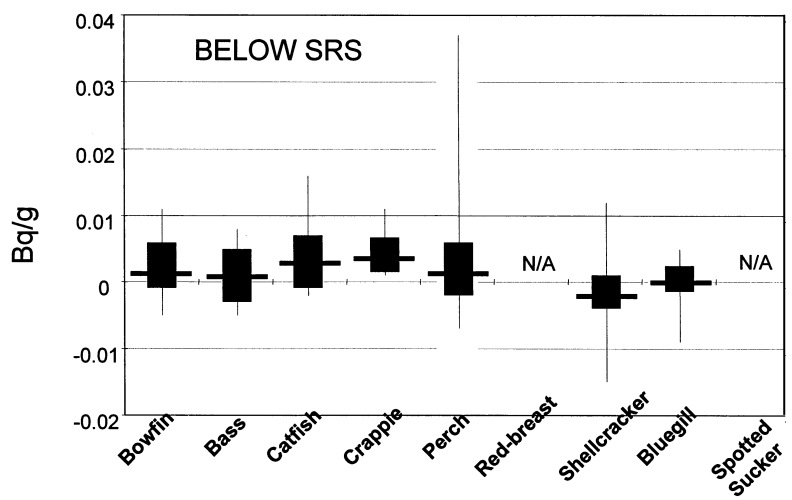

Fig. 2. Concentrations of radiocesium in fish muscle fillets (wet weight) collected from the locations on the Savannah River above, along, and below the U.S. Department of Energy's Savannah River Site (SRS). Vertical lines represent ranges, boxes represent $25 \%$ to $75 \%$ quartile limits, and horizontal lines represent the geometric means.

Table IV. Conversion of $\mathrm{Bq} / \mathrm{q}$ to $\mathrm{pCi} / \mathrm{g}$ for the Risk Assessment

\begin{tabular}{|c|c|c|c|c|c|c|c|c|}
\hline \multirow[b]{2}{*}{ Fish species } & \multicolumn{2}{|c|}{ Savannah River } & \multicolumn{2}{|c|}{ Steel Creek } & \multicolumn{2}{|c|}{ Savannah River } & \multicolumn{2}{|c|}{ Steel Creek } \\
\hline & $\begin{array}{c}\text { Arithmetic } \\
\text { mean } \\
(\mathrm{Bq} / \mathrm{g})\end{array}$ & $\begin{array}{c}\text { 95th } \\
\text { percentile } \\
(\mathrm{Bq} / \mathrm{g})\end{array}$ & $\begin{array}{c}\text { Arithmetic } \\
\text { mean } \\
(\mathrm{Bq} / \mathrm{g})\end{array}$ & $\begin{array}{c}\text { 95th } \\
\text { percentile } \\
(\mathrm{Bq} / \mathrm{g})\end{array}$ & $\begin{array}{c}\text { Arithmetic } \\
\text { mean } \\
(\mathrm{pCi} / \mathrm{g})\end{array}$ & $\begin{array}{c}\text { 95th } \\
\text { percentile } \\
(\mathrm{pCi} / \mathrm{g})\end{array}$ & $\begin{array}{c}\text { Arithmetic } \\
\text { mean } \\
(\mathrm{pCi} / \mathrm{g})\end{array}$ & $\begin{array}{c}\text { 95th } \\
\text { percentile } \\
(\mathrm{pCi} / \mathrm{g})\end{array}$ \\
\hline Bowfin & 0.009 & 0.056 & 0.060 & 0.141 & 0.243 & 1.512 & 1.620 & 3.807 \\
\hline Largemouth bass & 0.0037 & 0.012 & 0.075 & 0.183 & 0.100 & 0.324 & 2.025 & 4.941 \\
\hline Channel catfish & 0.0037 & 0.016 & 0.069 & 0.141 & 0.100 & 0.432 & 1.863 & 3.807 \\
\hline Black crappie & 0.0032 & 0.017 & 0.012 & 0.024 & 0.086 & 0.459 & 0.324 & 0.648 \\
\hline Yellow perch & 0.004 & 0.027 & 0.053 & 0.143 & 0.108 & 0.729 & 1.431 & 3.861 \\
\hline Shellcracker & 0.003 & 0.018 & na & na & 0.081 & 0.486 & 0.000 & na \\
\hline Red-breasted sunfish & 0.002 & 0.014 & 0.069 & 0.160 & 0.054 & 0.378 & 1.863 & 4.320 \\
\hline Bluegill & 0.008 & 0.013 & na & na & 0.216 & 0.351 & 0.000 & na \\
\hline Spotted sucker & 0.002 & 0.008 & 0.021 & 0.051 & 0.054 & 0.216 & 0.567 & 1.377 \\
\hline
\end{tabular}

Note: $1 \mathrm{~Bq}=27 \mathrm{pCi} ; \mathrm{na}=$ not applicable (species not obtained in Steel Creek). 
Table V. Annual and Daily Fish Consumption for Fishermen along the Savannah River

\begin{tabular}{|c|c|c|c|c|c|c|}
\hline & $\begin{array}{l}\text { Arithmetic } \\
\text { mean }\end{array}$ & Median & $\begin{array}{c}\text { 75th } \\
\text { percentile }\end{array}$ & $\begin{array}{c}\text { 90th } \\
\text { percentile }\end{array}$ & $\begin{array}{c}\text { 95th } \\
\text { percentile }\end{array}$ & Maximum \\
\hline \multicolumn{7}{|c|}{ Consumption (kg/year) } \\
\hline Black males & 25.6 & 18.9 & 48.0 & 62.7 & 68.8 & 96.0 \\
\hline White males & 14.0 & 6.9 & 19.5 & 28.8 & 49.4 & 114.5 \\
\hline \multicolumn{7}{|c|}{ Consumption (g/day) } \\
\hline Black males & 70.1 & 51.8 & 131.5 & 171.8 & 187.9 & 263.0 \\
\hline White males & 38.4 & 18.8 & 53.4 & 78.9 & 135.3 & 313.7 \\
\hline
\end{tabular}

Note: Based on data from Burger et al. ${ }^{(10)}$

trophic levels, with piscivores generally having higher levels than those fish that feed lower on the food chain.

Although the overall sample of fish from the Savannah River showed a positive correlation between ${ }^{137} \mathrm{Cs}$ levels and body mass $(r=0.24, N=435, p<$ 0.0001 , Table III), this relation within species was not consistent. Although fish mass is often a surrogate for age and larger, older individuals tend to have higher contaminant levels, low correlations were found for most species. Indeed, the correlation was negative only for the species, bowfin, that demonstrated a sta- tistically significant relation. Two species, catfish and shellcracker, showed marginally significant $(p=0.07)$ positive correlations.

\subsection{Risk Estimates}

The conversion of $\mathrm{Bq} / \mathrm{g}$ (the standard international system) to $\mathrm{pCi} / \mathrm{g}$ for risk estimates was necessary (shown in Table IV, because each of these two measurements is used by different risk assessors and in different publications, and the slope factor is ex-

Table VI. Annual ${ }^{137} \mathrm{Cs}$ Dose and Incremental Cancer Risk from Consuming Each Species of Fish from the Savannah River

\begin{tabular}{|c|c|c|c|c|c|c|c|c|c|}
\hline \multirow[b]{2}{*}{ Fish species } & \multirow{2}{*}{$\begin{array}{c}{ }^{137} \mathrm{Cs} \\
\text { arithmetic } \\
\text { mean } \\
(\mathrm{pCi} / \mathrm{g})\end{array}$} & \multicolumn{4}{|c|}{ Black Males } & \multicolumn{4}{|c|}{ White Males } \\
\hline & & Median & $\begin{array}{c}\text { 75th } \\
\text { percentile } \\
(48 \mathrm{~kg} / \mathrm{yr})\end{array}$ & $\begin{array}{c}\text { 95th } \\
\text { percentile } \\
(68.8 \mathrm{~kg} / \mathrm{yr})\end{array}$ & $\begin{array}{l}\text { Maximum } \\
(96 \mathrm{~kg} / \mathrm{yr})\end{array}$ & $\begin{array}{l}\text { Median } \\
(6.9 \mathrm{~kg} / \mathrm{yr})\end{array}$ & $\begin{array}{c}\text { 75th } \\
\text { percentile } \\
(19.5 \mathrm{~kg} / \mathrm{yr})\end{array}$ & $\begin{array}{c}\text { 95th } \\
\text { percentile } \\
(49.4 \mathrm{~kg} / \mathrm{yr})\end{array}$ & $\begin{array}{l}\text { Maximum } \\
(114.5 \mathrm{~kg} / \mathrm{yr})\end{array}$ \\
\hline $\begin{array}{l}\text { Bowfin (pCi/year) } \\
\text { Risk }\end{array}$ & 0.243 & $\begin{array}{c}4593 \\
1.5 \mathrm{E}-07\end{array}$ & $\begin{array}{c}11664 \\
3.7 \mathrm{E}-07\end{array}$ & $\begin{array}{c}16718 \\
5.3 \mathrm{E}-07\end{array}$ & $\begin{array}{c}23328 \\
7.4 \mathrm{E}-07\end{array}$ & $\begin{array}{c}1677 \\
5.3 \mathrm{E}-08\end{array}$ & $\begin{array}{c}4739 \\
1.5 \mathrm{E}-07\end{array}$ & $\begin{array}{c}12004 \\
3.8 \mathrm{E}-07\end{array}$ & $\begin{array}{c}27824 \\
8.8 \mathrm{E}-07\end{array}$ \\
\hline $\begin{array}{l}\text { Largemouth bass }(\mathrm{pCi} / \mathrm{yr}) \\
\text { Risk }\end{array}$ & 0.100 & $\begin{array}{c}1888 \\
6.0 \mathrm{E}-08\end{array}$ & $\begin{array}{c}4795 \\
1.5 \mathrm{E}-07\end{array}$ & $\begin{array}{c}6873 \\
2.2 \mathrm{E}-07\end{array}$ & $\begin{array}{c}9590 \\
3.0 \mathrm{E}-07\end{array}$ & $\begin{array}{c}689 \\
2.2 \mathrm{E}-08\end{array}$ & $\begin{array}{c}1948 \\
6.2 \mathrm{E}-08\end{array}$ & $\begin{array}{c}4935 \\
1.6 \mathrm{E}-07\end{array}$ & $\begin{array}{c}11439 \\
3.6 \mathrm{E}-07\end{array}$ \\
\hline $\begin{array}{l}\text { Channel catfish (pCi/yr) } \\
\text { Risk }\end{array}$ & 0.100 & $\begin{array}{c}1888 \\
6.0 \mathrm{E}-08\end{array}$ & $\begin{array}{c}4795 \\
1.5 \mathrm{E}-07\end{array}$ & $\begin{array}{c}6873 \\
2.2 \mathrm{E}-07\end{array}$ & $\begin{array}{c}9590 \\
3.0 \mathrm{E}-07\end{array}$ & $\begin{array}{c}689 \\
2.2 \mathrm{E}-08\end{array}$ & $\begin{array}{c}1948 \\
6.2 \mathrm{E}-08\end{array}$ & $\begin{array}{c}4935 \\
1.6 \mathrm{E}-07\end{array}$ & $\begin{array}{c}11439 \\
3.6 \mathrm{E}-07\end{array}$ \\
\hline $\begin{array}{l}\text { Black crappie (pCi/yr) } \\
\text { Risk }\end{array}$ & 0.086 & $\begin{array}{c}1633 \\
5.2 \mathrm{E}-08\end{array}$ & $\begin{array}{c}4147 \\
1.3 \mathrm{E}-07\end{array}$ & $\begin{array}{c}5944 \\
1.9 \mathrm{E}-07\end{array}$ & $\begin{array}{c}8294 \\
2.6 \mathrm{E}-07\end{array}$ & $\begin{array}{c}596 \\
1.9 \mathrm{E}-08\end{array}$ & $\begin{array}{c}1685 \\
5.3 \mathrm{E}-08\end{array}$ & $\begin{array}{c}4268 \\
1.3 \mathrm{E}-07\end{array}$ & $\begin{array}{c}9893 \\
3.1 \mathrm{E}-07\end{array}$ \\
\hline $\begin{array}{l}\text { Yellow perch (pCi/yr) } \\
\text { Risk }\end{array}$ & 0.108 & $\begin{array}{c}2041 \\
6.5 \mathrm{E}-08\end{array}$ & $\begin{array}{c}5184 \\
1.6 \mathrm{E}-07\end{array}$ & $\begin{array}{c}7430 \\
2.3 \mathrm{E}-07\end{array}$ & $\begin{array}{c}10368 \\
3.3 \mathrm{E}-07\end{array}$ & $\begin{array}{c}745 \\
2.4 \mathrm{E}-08\end{array}$ & $\begin{array}{c}2106 \\
6.7 \mathrm{E}-08\end{array}$ & $\begin{array}{c}5335 \\
1.7 \mathrm{E}-07\end{array}$ & $\begin{array}{c}12366 \\
3.9 \mathrm{E}-07\end{array}$ \\
\hline $\begin{array}{l}\text { Shellcracker (pCi/yr) } \\
\text { Risk }\end{array}$ & 0.081 & $\begin{array}{c}1531 \\
4.8 \mathrm{E}-08\end{array}$ & $\begin{array}{c}3888 \\
1.2 \mathrm{E}-07\end{array}$ & $\begin{array}{c}5573 \\
1.8 \mathrm{E}-07\end{array}$ & $\begin{array}{c}7776 \\
2.5 \mathrm{E}-07\end{array}$ & $\begin{array}{c}559 \\
1.8 \mathrm{E}-08\end{array}$ & $\begin{array}{c}1580 \\
5.0 \mathrm{E}-08\end{array}$ & $\begin{array}{c}4001 \\
1.3 \mathrm{E}-07\end{array}$ & $\begin{array}{c}9275 \\
2.9 \mathrm{E}-07\end{array}$ \\
\hline $\begin{array}{l}\text { Red-breasted sunfish (pCi/yr) } \\
\text { Risk }\end{array}$ & 0.054 & $\begin{array}{c}1021 \\
3.2 \mathrm{E}-08\end{array}$ & $\begin{array}{c}2592 \\
8.2 \mathrm{E}-08\end{array}$ & $\begin{array}{c}3715 \\
1.2 \mathrm{E}-07\end{array}$ & $\begin{array}{c}5184 \\
1.6 \mathrm{E}-07\end{array}$ & $\begin{array}{c}373 \\
1.2 \mathrm{E}-08\end{array}$ & $\begin{array}{c}1053 \\
3.3 \mathrm{E}-08\end{array}$ & $\begin{array}{c}2668 \\
8.4 \mathrm{E}-08\end{array}$ & $\begin{array}{c}6183 \\
2.0 \mathrm{E}-07\end{array}$ \\
\hline $\begin{array}{l}\text { Bluegill }(\mathrm{pCi} / \mathrm{yr}) \\
\text { Risk }\end{array}$ & 0.022 & $\begin{array}{c}408 \\
1.3 \mathrm{E}-08\end{array}$ & $\begin{array}{c}1037 \\
3.3 \mathrm{E}-08\end{array}$ & $\begin{array}{c}1486 \\
4.7 \mathrm{E}-08\end{array}$ & $\begin{array}{c}2074 \\
6.6 \mathrm{E}-08\end{array}$ & $\begin{array}{c}149 \\
4.7 \mathrm{E}-09\end{array}$ & $\begin{array}{c}421 \\
1.3 \mathrm{E}-08\end{array}$ & $\begin{array}{c}1067 \\
3.4 \mathrm{E}-08\end{array}$ & $\begin{array}{c}2473 \\
7.8 \mathrm{E}-08\end{array}$ \\
\hline $\begin{array}{l}\text { Spotted sucker }(\mathrm{pCi} / \mathrm{yr}) \\
\text { Risk }\end{array}$ & 0.054 & $\begin{array}{c}1021 \\
3.2 \mathrm{E}-08\end{array}$ & $\begin{array}{c}2592 \\
8.2 \mathrm{E}-08\end{array}$ & $\begin{array}{c}3715 \\
1.2 \mathrm{E}-07\end{array}$ & $\begin{array}{c}5184 \\
1.6 \mathrm{E}-07\end{array}$ & $\begin{array}{c}373 \\
1.2 \mathrm{E}-08\end{array}$ & $\begin{array}{c}1053 \\
3.3 \mathrm{E}-08\end{array}$ & $\begin{array}{c}2668 \\
8.4 \mathrm{E}-08\end{array}$ & $\begin{array}{c}6183 \\
2.0 \mathrm{E}-07\end{array}$ \\
\hline $\begin{array}{l}\text { All species equally (pCi/yr) } \\
\text { Risk }\end{array}$ & 0.094 & $\begin{array}{c}1780 \\
5.6 \mathrm{E}-08\end{array}$ & $\begin{array}{c}4522 \\
1.4 \mathrm{E}-07\end{array}$ & $\begin{array}{c}6481 \\
2.0 \mathrm{E}-07\end{array}$ & $\begin{array}{c}9043 \\
2.9 \mathrm{E}-07\end{array}$ & $\begin{array}{c}650 \\
2.1 \mathrm{E}-08\end{array}$ & $\begin{array}{c}1837 \\
5.8 \mathrm{E}-08\end{array}$ & $\begin{array}{c}4653 \\
1.5 \mathrm{E}-07\end{array}$ & $\begin{array}{c}10786 \\
3.4 \mathrm{E}-07\end{array}$ \\
\hline
\end{tabular}

Note: Values were derived using consumption data for males shown in Table $\mathrm{V}$ and mean ${ }^{137} \mathrm{Cs}$ concentrations.

${ }^{a}$ Cancer risk slope factor for ${ }^{137} \mathrm{Cs}=3.2 \mathrm{E}-11 / \mathrm{pCi}$. 
pressed in $\mathrm{pCi} / \mathrm{g}$. Site-specific data on fish consumption in $\mathrm{kg} /$ year and $\mathrm{g} /$ day were used for Black and White males fishing along the Savannah River (after Burger et al. ${ }^{(10)}$ Table V). Both the arithmetic mean and the median for Black males were significantly higher than for White males. ${ }^{(10)}$ The risk for Black and White fishermen was also compared for a range of consumption levels (75th, 95th, and maximum; see Table V).

The intake calculations and the risk estimates from ${ }^{137} \mathrm{Cs}$ at the mean concentration in each of the fish species from the Savannah River (Table VI) and Steel Creek (Table VII) differed; risk was considerably higher for fish from Steel Creek. Further, because of the higher levels of fish consumption, Black males were more at risk than White males (Tables VI and VII)

Using mean ${ }^{137} \mathrm{Cs}$ concentrations and median fish consumption for 70 years for Black males, the excess lifetime risk associated with the nine species of fish in the Savannah River ranged from $9.0 \times 10^{-7}$ to $1.0 \times$ $10^{-5}$ (Fig. 3). The same calculation for the seven species of fish from Steel Creek ranged from 1.4 to $8.0 \times$ $10^{-5}$ for median consumption by Black males. The 95th percentile of fish consumption for Black males was about $70 \mathrm{~kg} /$ year (Table $\mathrm{V}$ ), yielding a range of risks shown in Tables VI and VII. Black fishermen consuming the $95 \%$ of only bass from Steel Creek would sustain a lifetime risk of $3.1 \times 10^{-4}$, whereas the same consumption of Savannah River bass would yield a risk estimate of $1.5 \times 10^{-5}$ (Fig. 3 ).

\section{DISCUSSION}

\subsection{Locational Differences}

One important question about the risk to ecosystem receptors (including humans) from industrial complexes is whether the levels of contaminants in fish or other organisms are higher only downstream from the industrial site, or whether levels may also be elevated upstream as well. Few studies, however, examine this question in a suite of fish species that include different trophic levels and movement/migratory habits. Further, there is considerable public interest in whether ${ }^{137} \mathrm{Cs}$ levels differ above, along, or below sites such as the SRS, in part because of potential human health risks from consumption of fish, but also because of the potential risk to other consumers and to the fish

Table VII. Annual ${ }^{137}$ Cs Dose and Incremental Cancer Risk ${ }^{a}$ from Consuming Each Species of Fish from Steel Creek

\begin{tabular}{|c|c|c|c|c|c|c|c|c|c|}
\hline \multirow[b]{2}{*}{ Fish species } & \multirow{2}{*}{$\begin{array}{c}{ }^{137} \mathrm{Cs} \\
\text { arithmetic } \\
\text { mean } \\
(\mathrm{pCi} / \mathrm{g})\end{array}$} & \multicolumn{4}{|c|}{ Black Males } & \multicolumn{4}{|c|}{ White Males } \\
\hline & & $\begin{array}{c}\text { Median } \\
(18.9 \mathrm{~kg} / \mathrm{yr})\end{array}$ & $\begin{array}{c}75 \text { th } \\
\text { percentile } \\
(48 \mathrm{~kg} / \mathrm{yr})\end{array}$ & $\begin{array}{c}95 \text { th } \\
\text { percentile } \\
(68.8 \mathrm{~kg} / \mathrm{yr})\end{array}$ & $\begin{array}{l}\text { Maximum } \\
(96 \mathrm{~kg} / \mathrm{yr})\end{array}$ & $\begin{array}{l}\text { Median } \\
(6.9 \mathrm{~kg} / \mathrm{yr})\end{array}$ & $\begin{array}{c}\text { 75th } \\
\text { percentile } \\
(19.5 \mathrm{~kg} / \mathrm{yr})\end{array}$ & $\begin{array}{c}\text { 95th } \\
\text { percentile } \\
(49.4 \mathrm{~kg} / \mathrm{yr})\end{array}$ & $\begin{array}{l}\text { Maximum } \\
(114.5 \mathrm{~kg} / \mathrm{yr})\end{array}$ \\
\hline $\begin{array}{l}\text { Bowfin }(\mathrm{pCi} / \mathrm{yr}) \\
\text { Risk }\end{array}$ & 1.620 & $\begin{array}{c}30618 \\
9.7 \mathrm{E}-07\end{array}$ & $\begin{array}{c}77760 \\
2.5 \mathrm{E}-06\end{array}$ & $\begin{array}{l}111456 \\
3.5 \mathrm{E}-06\end{array}$ & $\begin{array}{l}155520 \\
4.9 \mathrm{E}-06\end{array}$ & $\begin{array}{c}11178 \\
3.5 \mathrm{E}-07\end{array}$ & $\begin{array}{c}31590 \\
1.0 \mathrm{E}-06\end{array}$ & $\begin{array}{c}80028 \\
2.5 \mathrm{E}-06\end{array}$ & $\begin{array}{l}185490 \\
5.9 \mathrm{E}-06\end{array}$ \\
\hline $\begin{array}{l}\text { Largemouth bass }(\mathrm{pCi} / \mathrm{yr}) \\
\text { Risk }\end{array}$ & 2.025 & $\begin{array}{r}38273 \\
1.2 \mathrm{E}-06\end{array}$ & $\begin{array}{c}97200 \\
3.1 \mathrm{E}-06\end{array}$ & $\begin{array}{l}139320 \\
4.4 \mathrm{E}-06\end{array}$ & $\begin{array}{l}194400 \\
6.1 \mathrm{E}-06\end{array}$ & $\begin{array}{c}13973 \\
4.4 \mathrm{E}-07\end{array}$ & $\begin{array}{c}39488 \\
1.2 \mathrm{E}-06\end{array}$ & $\begin{array}{l}100035 \\
3.2 \mathrm{E}-06\end{array}$ & $\begin{array}{l}231863 \\
7.3 \mathrm{E}-06\end{array}$ \\
\hline $\begin{array}{l}\text { Channel catfish (pCi/yr) } \\
\text { Risk (pCi/yr) }\end{array}$ & 1.863 & $\begin{array}{r}35211 \\
1.1 \mathrm{E}-06\end{array}$ & $\begin{array}{c}89424 \\
2.8 \mathrm{E}-06\end{array}$ & $\begin{array}{l}128174 \\
4.1 \mathrm{E}-06\end{array}$ & $\begin{array}{l}178848 \\
5.7 \mathrm{E}-06\end{array}$ & $\begin{array}{c}12855 \\
4.1 \mathrm{E}-07\end{array}$ & $\begin{array}{c}36329 \\
1.1 \mathrm{E}-06\end{array}$ & $\begin{array}{c}92032 \\
2.9 \mathrm{E}-06\end{array}$ & $\begin{array}{l}213314 \\
6.7 \mathrm{E}-06\end{array}$ \\
\hline $\begin{array}{l}\text { Black crappie (pCi/yr) } \\
\text { Risk }\end{array}$ & 0.324 & $\begin{array}{r}6124 \\
1.9 \mathrm{E}-07\end{array}$ & $\begin{array}{c}15552 \\
4.9 \mathrm{E}-07\end{array}$ & $\begin{array}{c}22291 \\
7.0 \mathrm{E}-07\end{array}$ & $\begin{array}{r}31104 \\
9.8 \mathrm{E}-07\end{array}$ & $\begin{array}{c}2236 \\
7.1 \mathrm{E}-08\end{array}$ & $\begin{array}{c}6318 \\
2.0 \mathrm{E}-07\end{array}$ & $\begin{array}{c}16006 \\
5.1 \mathrm{E}-07\end{array}$ & $\begin{array}{r}37098 \\
1.2 \mathrm{E}-06\end{array}$ \\
\hline $\begin{array}{l}\text { Yellow perch }(\mathrm{pCi} / \mathrm{yr}) \\
\text { Risk }\end{array}$ & 1.431 & $\begin{array}{r}27046 \\
8.5 \mathrm{E}-07\end{array}$ & $\begin{array}{c}68688 \\
2.2 \mathrm{E}-06\end{array}$ & $\begin{array}{c}98453 \\
3.1 \mathrm{E}-06\end{array}$ & $\begin{array}{l}137376 \\
4.3 \mathrm{E}-06\end{array}$ & $\begin{array}{c}9874 \\
3.1 \mathrm{E}-07\end{array}$ & $\begin{array}{c}27905 \\
8.8 \mathrm{E}-07\end{array}$ & $\begin{array}{c}70691 \\
2.2 \mathrm{E}-06\end{array}$ & $\begin{array}{l}163850 \\
5.2 \mathrm{E}-06\end{array}$ \\
\hline Shellcracker & This specie & es was not ob & tained in $\mathrm{S}$ & eel Creek & & & & & \\
\hline $\begin{array}{l}\text { Red-breasted sunfish (pCi/yr) } \\
\text { Risk }\end{array}$ & 1.863 & $\begin{array}{c}35211 \\
1.1 \mathrm{E}-06\end{array}$ & $\begin{array}{c}89424 \\
2.8 \mathrm{E}-06\end{array}$ & $\begin{array}{l}128174 \\
4.1 \mathrm{E}-06\end{array}$ & $\begin{array}{l}178848 \\
5.7 \mathrm{E}-06\end{array}$ & $\begin{array}{c}12855 \\
4.1 \mathrm{E}-07\end{array}$ & $\begin{array}{c}36329 \\
1.1 \mathrm{E}-06\end{array}$ & $\begin{array}{c}92032 \\
2.9 \mathrm{E}-06\end{array}$ & $\begin{array}{l}213314 \\
6.7 \mathrm{E}-06\end{array}$ \\
\hline Bluegill & This specie & es was not ob & tained in $\mathrm{S}$ & el Creek & & & & & \\
\hline $\begin{array}{l}\text { Spotted sucker }(\mathrm{pCi} / \mathrm{yr}) \\
\text { Risk }\end{array}$ & 0.621 & $\begin{array}{r}11737 \\
3.7 \mathrm{E}-07\end{array}$ & $\begin{array}{c}29808 \\
9.4 \mathrm{E}-07\end{array}$ & $\begin{array}{c}42725 \\
1.4 \mathrm{E}-06\end{array}$ & $\begin{array}{r}59616 \\
1.9 \mathrm{E}-06\end{array}$ & $\begin{array}{c}4285 \\
1.4 \mathrm{E}-07\end{array}$ & $\begin{array}{c}12110 \\
3.8 \mathrm{E}-07\end{array}$ & $\begin{array}{c}30677 \\
9.7 \mathrm{E}-07\end{array}$ & $\begin{array}{r}71105 \\
2.2 \mathrm{E}-06\end{array}$ \\
\hline $\begin{array}{l}\text { All species equally (pCi/yr) } \\
\text { Risk }\end{array}$ & 1.083 & $\begin{array}{r}20469 \\
6.5 \mathrm{E}-07\end{array}$ & $\begin{array}{c}51984 \\
1.6 \mathrm{E}-06\end{array}$ & $\begin{array}{c}74510 \\
2.4 \mathrm{E}-06\end{array}$ & $\begin{array}{l}103968 \\
3.3 \mathrm{E}-06\end{array}$ & $\begin{array}{c}7473 \\
2.4 \mathrm{E}-07\end{array}$ & $\begin{array}{c}21119 \\
6.7 \mathrm{E}-07\end{array}$ & $\begin{array}{c}53500 \\
1.7 \mathrm{E}-06\end{array}$ & $\begin{array}{l}124004 \\
3.9 \mathrm{E}-06\end{array}$ \\
\hline
\end{tabular}

Note: Values were derived using the annual consumption data for males shown in Table V.

${ }^{a}$ Cancer risk slope factor for ${ }^{137} \mathrm{Cs}=3.2 \mathrm{E}-11 / \mathrm{pCi}$. 


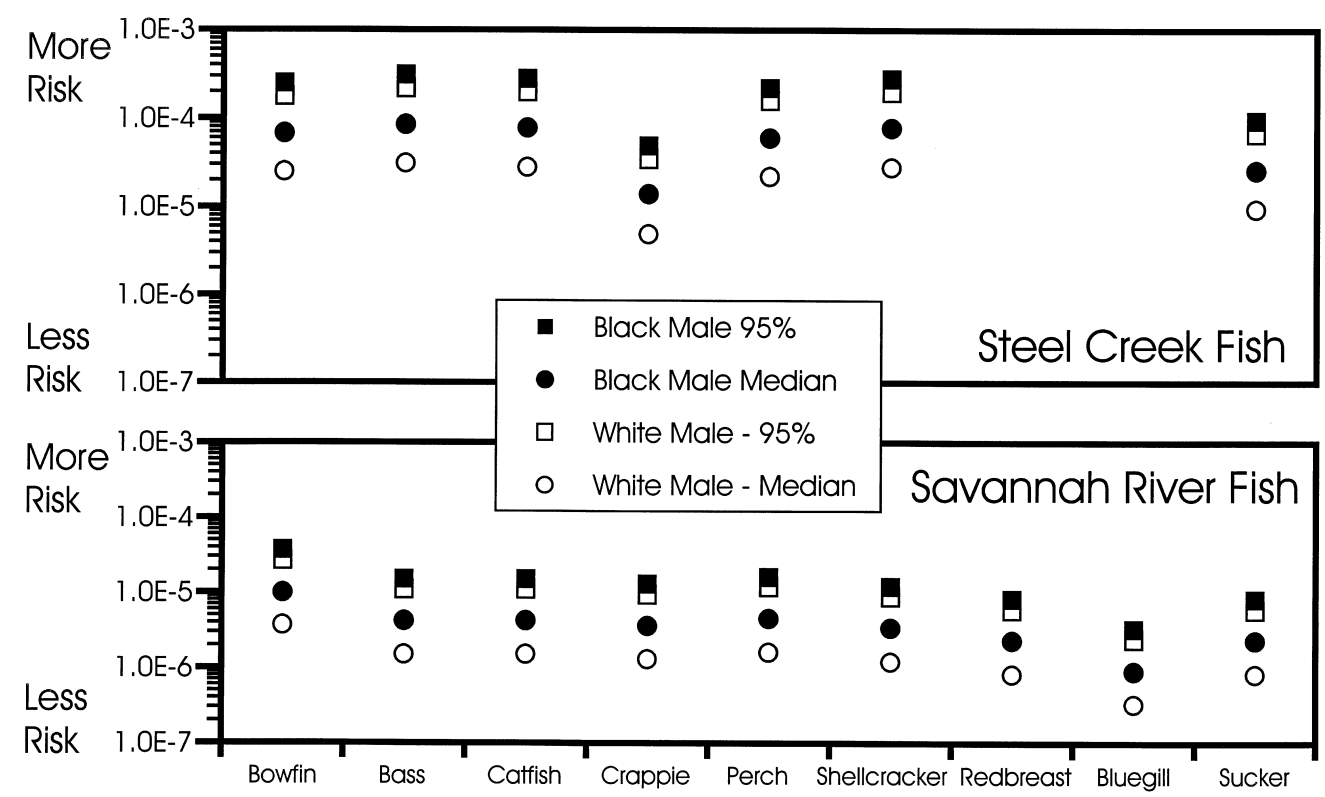

Fig. 3. Cancer risk from consumption of fish, based on site-specific consumption patterns at the median and $95 \%$ levels, for different fish (based on site-specific mean radiocesium levels). The public may consume fish from the Savannah River, but are not allowed to catch fish in Steel Creek.

themselves. However, the results of this study indicate few significant differences among species of fish collected from these three locations, except for bowfin and shellcracker, the former being significantly higher when collected from above the site. Catfish collected from above the site also showed higher levels, but the differences were not great. The lack of a difference above, along, and below the SRS is not surprising because the distances are not great, there are no barriers to impede free movement of fish along the river, and most of the species move within this stretch of the river. ${ }^{(32)}$ Only the sunfish and perch are sedentary. ${ }^{(32)}$

There were, however, significant locational differences in the ${ }^{137} \mathrm{Cs}$ levels in the muscle of seven species that were collected in Steel Creek versus the Savannah River. Levels in the muscle of fish from Steel Creek were an order of magnitude greater than levels in those fish collected from the Savannah River. This is not surprising, since Steel Creek is known to have been contaminated with ${ }^{137} \mathrm{Cs}$ when there was leakage from defective experimental fuel assemblages in the 1960s and 1970s. ${ }^{(31)}$ In the early 1960s, both P and L reactors discharged cooling water into Steel Creek. The pattern of releases differed among the different reactors on SRS, with the largest and latest releases occurring from $\mathrm{P}$ and $\mathrm{C}$ reactors (see Fig. 1; Paller et $\left.a l .{ }^{(34)}\right)$ Nonetheless, some releases occurred from $\mathrm{L}$ reactor until 1989, and during this period radionuclides continued to be released into the upper portion of Steel Creek.

All reactors on the SRS are presently closed down, and no new releases have occurred since $1989 .{ }^{(34)}$ However, the continuous erosion of contaminated sediments downstream into the lower reaches of Steel Creek keeps the levels of ${ }^{137} \mathrm{Cs}$ from falling. ${ }^{(35)}$ In a detailed study of reactor cooling reservoirs on the SRS, Whicker et al. ${ }^{(8)}$ found that most of the ${ }^{137} \mathrm{Cs}$ resided in the sediments, rather than the water column. Levels were highest in the top $6 \mathrm{~cm}$ of sediments, and declined markedly further down. ${ }^{137} \mathrm{Cs}$ is available to the algae, macrophytes, and benthic invertebrates in Steel Creek, and continues to circulate among the different compartments of the creek ecosystem, from sediments to biota. Thus the ecological half-life can be longer in resident biota than the physical half-life of 30 years. Whicker et al. ${ }^{(8)}$ found that the concentration ratio (concentration in fish/concentration in water) for fish muscle from another SRS area rich in ${ }^{137} \mathrm{Cs}$ was $10^{4}$ overall, and the ratios increased as a function of trophic level. Loss from the system was slow, and mainly through periodic outflow of water and suspended materials, rather than biotic export. ${ }^{(8)}$ As with other aquatic systems on the SRS, ${ }^{(8)}$ the principal mechanisms of loss from the Steel Creek system is presumably from radioactive decay and periodic outflow of water and suspended ma- 
terials; biotic export is relatively inconsequential. This suggests that fish obtain their exposure from benthic organisms, which reflect the levels in sediment. This is shown in the ${ }^{137} \mathrm{Cs}$ levels in the fish from Steel Creek, compared with fish from the Savannah River. Bioavailability is also a function of water temperature; ${ }^{137} \mathrm{Cs}$ uptake increases with temperature. ${ }^{(24,36)}$ Because Steel Creek and associated marshes are shallower than the Savannah River, water temperatures are generally higher in the summer when fish are more active, which might also contribute to higher uptake of ${ }^{137} \mathrm{Cs}$; parts of the Steel Creek Delta are shaded, however.

Long-term data generated by Paller et $a l^{(34)}$ from three species of fish from reservoirs and streams of the SRS indicate that the highest ${ }^{137} \mathrm{Cs}$ levels are found in fish from Steel Creek, even when compared with fish from Par Pond, which was the cooling reservoir for $\mathrm{P}$ and $\mathrm{R}$ reactors. In their study, the ecological half-life of ${ }^{137} \mathrm{Cs}$ ranged from 3.2 to 16.7 years, depending on the species and location of fish, ${ }^{(34)} \mathrm{com}-$ pared with the radioactive half-life of 30 years. ${ }^{(30)}$ The biological half-life depends in part on the excretion rate. ${ }^{(37)}$ The biological half-life was longest for fish in the lower reaches of the stream, ${ }^{(34)}$ an area where people might fish, and where there is a greater chance that fish would move out into the Savannah River.

The levels of ${ }^{137} \mathrm{Cs}$ in fish, however, are not solely influenced by radioactive decay, but by continued exposure generated by resuspension and sediment dynamics, as well as atmospheric deposition. The normal processes of sedimentation and sequestration of radionuclides will decrease their bioavailability to fish and other organisms. Thus, remediation measures that result in disturbance of sediments have the potential to increase the bioavailability of ${ }^{137} \mathrm{Cs}$ to fish, thus increasing the risk to humans and others who eat the fish from these regions.

The higher levels of ${ }^{137} \mathrm{Cs}$ in fish from Steel Creek suggest that disruption of sediments in Steel Creek could further increase levels in fish, both within Steel Creek and downstream in the Savannah River. This is particularly true given that most ${ }^{137} \mathrm{Cs}$ is now in the top $5 \mathrm{~cm}$ at Steel Creek, compared with the top 20 $\mathrm{cm}$, as was the case in the early $1970 \mathrm{~s} .{ }^{(38)}$ Disruption would increase downstream transport, potentially increasing the levels in fish in the Savannah River. Paller et al. $^{(34)}$ also found significant correlations between ${ }^{137} \mathrm{Cs}$ levels in water and in fish.

In a 1995 study of yellow bullhead catfish (Ameiurus [Ictalurus] natalis) collected from Pond B, a former cooling reservoir associated with $\mathrm{R}$ reactor, McCreedy et al. ${ }^{(21)}$ found that ${ }^{137} \mathrm{Cs}$ levels in muscle increased until 3 years of age, but did not increase further. There were no differences by mass or gender. Mean ${ }^{137} \mathrm{Cs}$ levels ranged from $2.63 \mathrm{~Bq} / \mathrm{g}$ (at age 1 year) to $3.60 \mathrm{~Bq} / \mathrm{g}$ (at age 5 years), which is an order of magnitude higher than those found in this study for channel catfish from Steel Creek, and two orders of magnitude higher than those from the Savannah River (to convert these values to $\mathrm{pCi} / \mathrm{g}$, multiply by 27 ).

Data on ${ }^{137} \mathrm{Cs}$ levels in fish analyzed by the South Carolina Department of Health, Georgia Department of Natural Resources, and the SRS ${ }^{(9)}$ were similar to, or higher than, data generated in this study. They found average levels that ranged from 16 to $40 \mathrm{pCi} / \mathrm{g}$ (wet weight) in the Savannah River along the SRS, with lower levels upstream from SRS.(9) Thus, the levels found in this study were similar to those used by the respective states.

The higher levels of ${ }^{137} \mathrm{Cs}$ in fish from Steel Creek, compared with those from the Savannah River are mirrored in the levels in organisms that eat fish. For example, ${ }^{137} \mathrm{Cs}$ in raccoons (Procyon lotor) from Steel Creek (median of $0.146 \mathrm{~Bq} / \mathrm{g}$ ) is higher than raccoons from immediately off-site (median of $0.011 \mathrm{~Bq} / \mathrm{g}$ ). ${ }^{(12)}$

\subsection{Species Differences}

For both Steel Creek and the Savannah River, the relative order of ${ }^{137} \mathrm{Cs}$ levels in fish muscle was similar among species. In general, ${ }^{137} \mathrm{Cs}$ levels in bowfin (SRS) and bass (Steel Creek) were highest, followed by perch and catfish, then by crappie, shellcracker, red-breasted sunfish, and sucker, with bluegill having the lowest levels. Differences in ${ }^{137} \mathrm{Cs}$ could be due to differences in exposure, relative uptake, and excretion, as well as trophic-level considerations (Table I). Bowfin, bass, catfish, and pickerel eat primarily large fish, with a few invertebrates, and might be expected to accumulate higher levels from their food than fish that eat mainly invertebrates or plant material. However, ${ }^{137} \mathrm{Cs}$ does not always biomagnify in fish; Whicker et al. ${ }^{8}$ found the highest levels in a planktonic herbivore.

Exposure is a function of where fish live relative to the source of contamination, and to the levels in the food and water they consume. Both the river and creek experienced exposure through atmospheric deposition from global fallout in the past, ${ }^{(39)}$ as well as exposure from point sources, such as from activities of the SRS. Nuclear weapons testing has been the most significant anthropogenic source of radiocesium in the global environment. ${ }^{(30)}$ The Chernobyl accident was also severe enough to inject radiocesium into atmospheric circulation, resulting in global distribution. ${ }^{(30)}$

The amount of time that radionuclides remain in an 
ecosystem is affected by physical (deposition, radioactive decay), chemical (adsorption to sediments, changes in oxidative state), and biological factors (translocation from sediment to water and to biota $\left.{ }^{(34)}\right)$. Fish assimilate ${ }^{137} \mathrm{Cs}$ relatively rapidly, and eliminate it relatively slowly. ${ }^{(37)}$ Thus the contaminant remains in fish even after water concentrations are no longer detectable. ${ }^{(34)}$

Because atmospheric deposition should be relatively constant across our study sites, differences would be due to local contamination, in this case from previous accidental releases from the SRS activities (see above). Levels of ${ }^{137} \mathrm{Cs}$ in fish may thus reflect trophic-level differences, because some of the fish are primarily piscivores, whereas others eat primarily invertebrates or detritus (Table I). In general, ${ }^{137} \mathrm{Cs}$ reflected trophic levels for both the Savannah River and Steel Creek fish. Species that eat larger and higher trophic-level prey had higher ${ }^{137} \mathrm{Cs}$ levels in muscle than those that ate smaller prey that are lower on the food chain.

Bowfin was an exception for Steel Creek; levels of ${ }^{137} \mathrm{Cs}$ were higher in muscle of bass than bowfin (Table III). This may reflect the fact that bowfin spend more time near the mouth of the Creek, and perhaps in the river, than do bass, which prefer nonflowing waters $^{(40)}$ and move farther up the creek. Bowfin spawn in shallow, weedy waters, ${ }^{(32)}$ which are found at the mouth of Steel Creek. Collection site alone is not enough to understand the movement patterns and sites of exposure for fish.

\subsection{Risk to Fish and Other Wildlife}

Assessing risk to fish themselves is complex, in part because, in most natural situations, fish are exposed to multiple contaminants. For example, mercury is sufficiently elevated in fish from the Savannah River so that there are fish consumption advisories. ${ }^{(9)}$ Sugg et al. ${ }^{(41)}$ examined the association of DNA damage with concentrations of mercury and ${ }^{137} \mathrm{Cs}$ in fish from several places on the SRS. They found that increased concentrations of toxicants were related to increased DNA damage, as well as to body condition, but they could not determine whether the contaminants were a cause or were merely correlated with the damage, nor could they separate the contribution of mercury. Further, the threshold level for DNA damage was not determined for each toxicant.

\subsection{Risk to Humans}

There is considerable interest in determining the risk to humans from radionuclide exposure from De- partment of Energy facilities, such as the SRS. The usual pathways considered include inhalation, ingestion of foods raised at the site boundary, immersion in a gas plume at the site boundary, external exposure at the site boundary, and ingestion of water or fish from the Savannah River that were contaminated by site liquid releases and/or atmospheric deposition. ${ }^{(42)}$ Jannik,${ }^{(42)}$ in examining risk from consumption of fish from the Savannah River adjacent to the SRS, assumed that the maximally exposed individual would consume $19 \mathrm{~kg} /$ year of fish. He reasoned that because of its remote location, recreational fishermen, as opposed to subsistence fishermen, were the most reasonable receptors. ${ }^{(42)}$

Using this calculation, Jannik ${ }^{(42)}$ concluded that the most critical pathway for the maximally exposed individual was from fish consumption and drinking water ingestion, the former considered a "nontypical pathway." However, he concluded that as long as site operations remained constant, only tritium would drive a maximally exposed individual's risk above $1 \times$ $10^{-6}$. Although the Food and Drug Administration's and EPA's most conservative action level is $1 \times 10^{-6}$ excess lifetime cancer risk, ${ }^{(43)} \mathrm{Jannik}^{(42)}$ suggested that only if the calculated risk is greater than $1 \times 10^{-4}$ is some corrective action required.

Our previous studies have now added new information to this risk scenario. ${ }^{(10)}$ We interviewed people fishing along the Savannah River, from the Augusta lock and dam to the Route 301 bridge (Fig. 1), and found that mean consumption for Blacks fishing on the river was $25.5 \mathrm{~kg} /$ year, compared with $14.0 \mathrm{~kg} /$ year for Whites. Additionally, 35\% (72/203) of people interviewed said they ate more than $19 \mathrm{~kg} /$ year. Although it could be argued that some of these people did not specifically fish along the SRS, but fished the Savannah River above and below the SRS, the data presented in this article show that there is little difference in ${ }^{137} \mathrm{Cs}$ levels in fish captured above, along, or below the SRS. Both the fish collected and the consumption surveys were conducted in the same region. Thus, future risk assessments for ${ }^{137} \mathrm{Cs}$ should take into account two factors: (1) many people who fish in this stretch of river consume more than $19 \mathrm{~kg} /$ year, and (2) the levels of ${ }^{137} \mathrm{Cs}$ in fish fillets generally do not differ significantly whether the fish are captured above, along, or below the SRS.

None of the fish fillets from Steel Creek or the Savannah River exceeded the European Economic Community limit of $0.60 \mathrm{~Bq} / \mathrm{g}^{(44)}$; the highest level for an individual fish was $0.141 \mathrm{~Bq} / \mathrm{g}$, which was found in bowfin collected from Steel Creek. If fishermen consumed the median amount of fish $(18.9 \mathrm{~kg} /$ year for 
Black males), and took only bass (with mean concentrations of ${ }^{137} \mathrm{Cs}=0.075 \mathrm{~Bq} / \mathrm{g}$ ) from Steel Creek for 70 years, their risk would be $8.5 \times 10^{-5}$. Because fishing is not allowed along Steel Creek, it is reasonable to focus most attention on the Savannah River. However, there is some on-site subsistence poaching of fish.

Using mean ${ }^{137} \mathrm{Cs}$ concentrations and median fish consumption for 70 years for Black males, the excess lifetime risk associated with the eight species of fish in the Savannah River ranged from $9.0 \times 10^{-7}$ to $1.0 \times$ $10^{-5}$; and for Steel Creek, ranged from 1.4 to $8.0 \times$ $10^{-5}$. Black fishermen consuming the $95 \%$ level of bass from the Savannah River would sustain a lifetime risk of $1.5 \times 10^{-5}$, whereas the same consumption of Steel Creek bass would yield a risk estimate of $3.1 \times 10^{-4}$.

One factor often considered in risk reduction for humans consuming fish is varying either the species consumed or the size of the fish. In this study, there was a positive correlation overall between weight and ${ }^{137} \mathrm{Cs}$, indicating that eating smaller fish would result in lower exposure. Whicker et al. ${ }^{(8)}$ also found a correlation between weight and ${ }^{137} \mathrm{Cs}$ levels $(r=0.31)$ in fish from a former reactor cooling reservoir on the SRS, but there were no significant correlations for weight within species. Similarly, McCreedy et al. ${ }^{(21)}$ found no correlation between weight and ${ }^{137} \mathrm{Cs}$ for yellow bullhead catfish from the same cooling reservoir. When ${ }^{137} \mathrm{Cs}$ levels are high, as occurred following the Chernobyl accident, a strong linear relation is found between weight and ${ }^{137} \mathrm{Cs} .{ }^{(45)}$ However, in some studies, some fish species show a significant positive relation and others do not. ${ }^{(4)}$

In our study, the size-mass relation was not consistent for individual fish species (Table III). Bowfin and bass showed a negative correlation. Although the other relations between mass and levels of ${ }^{137} \mathrm{Cs}$ were all positive, they approached significance only for catfish and shellcracker. The reasons for lower levels in larger bowfin may relate to differences in location of foraging and prey; younger bowfin may feed on benthic organisms found on the bottom. In some fish (bluegill, among others), there is also a decrease in the rate of ${ }^{137} \mathrm{Cs}$ elimination with increasing weight, ${ }^{(8,24)}$ which would result in higher retention of ${ }^{137} \mathrm{Cs}$ in larger fish. This might also operate for the bowfin and bass, two of the largest fish species sampled in this study. An inverse relation between weight and levels of ${ }^{137} \mathrm{Cs}$ might also result from differences in mobility: If larger fish moved farther away at some times of the year, they might be exposed to lower ${ }^{137} \mathrm{Cs}$ levels away from the SRS. This hypothesis requires further study. It could be that the largest bass are in the reservoirs. ${ }^{(40)}$

Finally, it should be noted that consumption of fish contaminated with ${ }^{137} \mathrm{Cs}$ is only one route of exposure for the general public. People can also be exposed to ${ }^{137} \mathrm{Cs}$ through consumption of other wild game from the SRS (deer, wild hogs, squirrel, raccoon, and others ${ }^{(12,27,47)}$, from drinking water contaminated with ${ }^{137} \mathrm{Cs}$, and through inhalation of radionuclides. Both the fish from the Savannah River, and other foods that people consume also contain other contaminants, such as mercury, ${ }^{(48)}$ that should be considered in a cumulative risk assessment. In issuing consumption advisories, South Carolina mainly considered the risk from mercury, as well as from radionuclides. ${ }^{(9,26)}$ Other pathways of exposure, such as inhalation and dermal exposure of contaminants, must also be added to total cumulative risk.

\section{ACKNOWLEDGMENTS}

The authors thank C. Dixon, D. Kling, and C. Lord for field and laboratory assistance; and B. D. Goldstein, B. Friedlander, D. Kosson, P. Lioy, C. Powers, C. Schopfer, and A. Upton for valuable comments on the research and manuscript. This research was funded by the Consortium for Risk Evaluation with Stakeholder Participation (CRESP) through the Department of Energy (DOE; AI \#DE-FC01-95EW55084, DE-FG 26-00NT 40938), and by DOE contract \#DE-ACO976SR00819 with the University of Georgia. The results, conclusions, and interpretations reported herein are the sole responsibility of the authors, and should not in any way be interpreted as representing the views of the funding.

\section{REFERENCES}

1. Sheehan, P. J., Miller, D. R., Butler, G. C., \& Bourdeau, P. (1984). (Eds.). Effects of pollutants at the ecosystem level. Chichester, England: Wiley.

2. Brisbin, I. L., Jr. (1991). Avian radioecology. Current Ornithology, 8, 69-140.

3. Burger, J. (1993). Metals in avian feathers: Bioindicators of environmental pollution. Reviews in Environmental Toxicology, 5, 203-311.

4. Renzoni, A., Mattei, N., Lari, L., \& Fossi, M. C. (1994). Contaminants in the environment. Boca Raton, FL: Lewis.

5. Hoffman, D. J., Rattner, B. A., Burton, G. A., Jr., \& Cairns, J., Jr. (1995). Handbook of ecotoxicology. Boca Raton, FL: Lewis.

6. Linthurst, R. A., Bourdeau, P., \& Tardiff, R. G. (1995). Methods to assess the effects of chemicals on ecosystems. Chicester, England: Wiley.

7. Kolehmainen, S. E. (1972). The balance of ${ }^{137} \mathrm{Cs}$, stable cesium and potassium of bluegill (Lepomis macrochirus Raf.) and other fish in White Oak Lake. Health Physics, 23, 301-315.

8. Whicker, F. W., Pinder, J. E., III, Bowling, J. W., Alberts, J. J., \& Brisbin, I. L., Jr. (1990). Distribution of long-lived radionuclides in an abandoned reactor cooling reservoir. Ecological Monographs, 60, 471-496. 
9. South Carolina Department of Health and Environmental Control (SCDHEC). (1996). Public health evaluation: Cesium-137 and strontium-90 in fish (Attachment to the fish consumption advisory for the Savannah River; \#3-5/14/96). Columbia, SC: Author.

10. Burger, J., Stephens, W., Boring, C. S., Kuklinski, M., Gibbons, J. W., \& Gochfeld, M. (1999). Ethnic and socioeconomic differences in exposure from fish caught along the Savannah River. Risk Analysis, 19, 427-438.

11. Burger, J. (1998). Fishing and risk along the Savannah River: Possible intervention. Journal of Toxicology and Environmental Health, 55, 405-419.

12. Gaines, K. F., Lord, C. G., Boring, C. S., Brisbin, I. L., Jr., Gochfeld, M., \& Burger, J. (2000). Raccoons as a potential vector of radionuclide contaminations to human food chains from a nuclear industrial site. Journal of Wildlife Management, $64,199-208$

13. Shimada, Y., Morisawa, S., \& Inoue, Y. (1996). A numerical model for the analysis and evaluation of global ${ }^{137} \mathrm{Cs}$ fallout. Health Physics, 70,171-179.

14. Evans, D. W., Alberts, J. J., \& Clark, R. A. I. (1983). Reversible ion-exchange fixation of cesium-137 leading to mobilization from reservoir sediments. Geochimica et Cosmochimica Acta, 47,1041-1049.

15. Fendley, T. T., Manlove, M. N., \& Brisbin, I. L., Jr. (1977). The accumulation and elimination of radiocesium by naturally contaminated wood ducks. Health Physics, 32, 415-422.

16. Brisbin, I. L., Jr., \& Smith, M. H. (1975). Radiocesium concentrations in whole-body homogenates and several body compartments of naturally contaminated white-tailed deer. In F. G. Howell, J. B. Gentry, \& M. H. Smith (Eds.), Mineral cycling in southeastern ecosystems (pp. 542-556). Springfield, VA: National Technical Information Service.

17. Kennamer, R. A., Brisbin, I. L., Jr., McCreedy, C. D., \& Burger, J. (1998). Radiocesium in mourning doves foraging on the exposed lakebed of a contaminated reactor-cooling reservoir: Risk to human consumers and temporal effects of the draw-down. Journal of Wildlife Management, 62, 497508.

18. Narayanyan, N., \& Eapen, J. (1971). Gross and subcellular distribution of cesium-137 in pigeon (Columba livia) tissues with special reference to muscles. Journal of Radiation Research, $12,51-55$.

19. Potter, C. M., Brisbin, I. L., Jr., \& McDowell, S. G. (1989). Distribution of ${ }^{137} \mathrm{Cs}$ in the American Coot (Fulica americana). Journal of Environmental Radioactivity, 9, 105-115.

20. Peles, J. D., Philippi, T., Smith, M. H., Brisbin, I. L., Jr., \& Gibbons, J. W. (2000). Seasonal variation in radiocesium levels of largemouth bass (Micropterus salmoides): Implications for humans and sensitive wildlife species. Environmental Toxicology and Chemistry, 19, 1830-1836.

21. McCreedy, C. D., Jagoe, C. H., Glickman, L. T., \& Brisbin, I. L., Jr. (1997). Bioaccumulation of cesium-137 in yellow bullhead catfish (Ameiurus natalis) inhabiting an abandoned nuclear reactor reservoir. Environmental Toxicology and Chemistry, 16, 328-335.

22. Noshkin, V. E., Robison, W. L., Wong, K. M., Brunk, J. L., Eagle, R. J., \& Jones, H. E. (1997). Past and present levels of some radionuclides in fish from Bikini and Enewetak Atolls. Health Physics, 73, 49-65.

23. Hakanson, L., Andersson, T., \& Nilsson, A. (1989). Caesium137 in perch in Swedish lakes after Chernobyl-present situation, relationships and trends. Environmental Pollution, 58, 195-212.

24. Ugedal, O., Forseth, T., Jonsson, B., \& Njastad, O. (1995). Sources of variation in radiocesium levels between individual fish from a Chernobyl contaminated Norwegian lake. Journal of Applied Ecology, 32, 352-361.

25. Jagoe, C. H., Chesser, R. K., Smith, M. H., Lomakin, M. D.,
Lingenfelser, S. K., \& Dallas, C. E. (1997). Levels of cesium, mercury and lead in fish, and cesium in pond sediment in an inhabited region of the Ukraine near Chernobyl. Environmental Pollution, 98, 223-232.

26. South Carolina Department of Health and Environmental Control (SCDHEC). (1996). Review of data from SCDHEC, GDNR, and SRS regarding radionuclides in Savannah river fish. Aiken, SC: Author.

27. Burger, J. (1997). Recreation and risk: Potential exposure. Journal of Toxicology and Environmental Health, 52, 269284.

28. Workman, S. W., \& McLeod, K. W. (1990). Vegetation of the Savannah River Site: Major community types (Publication SRONERP-19). Aiken, SC: Savannah River Ecology Laboratory.

29. Cummins, C. L., Hetrick, C. S., \& Martin, D. K. (1991). Radioactive releases at the Savannah River Site 1954-1989 (Report WSRC-RP-91-684). Aiken, SC: Westinghouse Savannah River Company.

30. Carlton, W. H., Murphy, C. E., Jr., \& Evans, A. G. (1994). Radiocesium in the Savannah River Site environment. Health Physics, 67, 233-244.

31. Ashley, C., \& Zeigler, C. C. (1980). Releases of radioactivity at the Savannah River Plant, 1954 through 1978 (Report DPSPU 75-25-1). Aiken, SC: E. I. DuPont de Nemours and Company.

32. Rohde, F. C., Arndt, R. G., Lindquist, D. G., \& Parnell, J. F. (1994). Freshwater fishes of the Carolinas, Virginia, Maryland, and Delaware. Chapel Hill, NC: University of North Carolina Press.

33. Currie, L. A. (1968). Limits for qualitative detection and quantitative determination. Analytical Chemistry, 40, 586-593.

34. Paller, M. H., Littrell, J. W., \& Peters, E. L. (1999). Ecological half-lives of ${ }^{137} \mathrm{Cs}$ in fishes from the Savannah River Site. Health Physics, 77, 392-402.

35. Peters, E. L., \& Brisbin, I. L., Jr. (1996). Environmental influences on the ${ }^{137} \mathrm{Cs}$ kinetics of the yellow-bellied turtle (Trachyemys scripta). Ecological Monographs, 66, 115136.

36. Rowan, J. R., \& Rasmussen, J. B. (1994). Bioaccumulation of radiocesium by fish: The influence of physicochemical factors and trophic structure. Canadian Journal of Fisheries and Aquatic Science, 51, 2388-2410.

37. Rowan, J. R., \& Rasmussen, J. B. (1995). The elimination of radiocesium in fish. Journal of Applied Ecology, 32, 739-744.

38. Brisbin, I. L., Jr., Beyers, R. J., Dapson, R. W., Geiger, R. A., Gentry, J. B., Gibbons, J. W., Smith, M. H., \& Woods, S. K. (1974). Patterns of radiocesium in the sediments of a stream channel contaminated by production reactor effluents. Health Physics, 27, 19-27.

39. Santschi, P. H., Bollhalder, S., Zingg, S., Luck, A., \& Farrenkothen, K. (1990). The self-cleaning capacity of surface waters after radioactive fallout. Evidence from European waters after Chernobyl, 1986-1988. Environmental Science and Technology, 24, 519-527.

40. Bennett, D. H., \& McFarlane, R. W. (1983). The fishes of the Savannah River Plant: National Environmental Research Park (SRO-NERP-12). Aiken, SC: Savannah River Ecology Laboratory.

41. Sugg, D. W., Chesser, R. K., Brooks, J. A., \& Grasman, B. T. (1995). The association of DNA damage to concentrations of mercury and radiocesium in largemouth bass. Environmental Toxicology and Chemistry, 14, 661-668.

42. Jannik, G. T. (1999). Critical radionuclide/critical pathway analysis for the U.S. Department of Energy's Savannah River Site. Risk Analysis, 19, 417-426.

43. Rodericks, J. V. (1992). Calculated risks: The toxicity and human health risks of chemicals in our environment. Cambridge, U.K.: Cambridge University Press.

44. European Economic Community (EEC). (1986). Derived ref- 
erence levels as a basis for the control of foodstuffs following a nuclear accident: A recommendation from the group of experts set up under Article 31 of the Euratom Treaty (EEC Regulation 170/86). Brussels, Belgium: Commission of the EEC Printing Office.

45. Koulikov, A. O., \& Ryabov, I. N. (1992). Specific cesium activity in freshwater fish and the size effect. The Science of the Total Environment, 112, 125-142.

46. Elliott, J. M., Hilton, J., Rigg, E., Tullett, P. A., Swift, D. J., \&
Leonard, D. R. P. (1992). Sources of variation in post-Chernobyl radiocesium in fish from two Cumbrian lakes (north-west England). Journal of Applied Ecology, 29, 108-119.

47. Burger, J. (2000). Gender differences in meal patterns: Role of self-caught fish and wild game in meat and fish diets. Environmental Research, 83, 140-149.

48. Burger, J., Gaines, K. F., \& Gochfeld, M. (2001). Ethnic differences in risk from mercury among Savannah River fisherman. Risk Analysis, 21, 533-544. 